Article original

\title{
Facteurs affectant les épizooties de Entomophaga grylli (Zygomycètes, Entomophthorales) chez les populations du criquet Zonocerus variegatus au Sud du Bénin en Afrique de l'Ouest
}

\author{
Ayekpa J. GnAGo ${ }^{1 *}$, Kouahou Foua BI ${ }^{2}$ \& Kouassi A. BEKon ${ }^{1+}$ \\ 'Institut National Polytechnique Félix Houphouet Boigny (INP-HB) Yamoussoukro, BP 1313 Côte d'Ivoire \\ 2Université de Cocody, UFR Biosciences, BP 582 Abidjan, Côte d'Ivoire \\ * Auteur pour les correspondances (E-mail: gayekpa@yahoo.fr) \\ Reçu le 17-04-2008, accepté le 17-06-2009.
}

\begin{abstract}
Résumé
L'étude en champ des facteurs affectant les épizooties de Entomophaga grylli chez les populations de Zonocerus variegatus, dans les quatre départements au Sud du Bénin, a montré que E. grylli est l'un des principaux facteurs de mortalité des larves âgées du criquet. Les infections ont lieu principalement de novembre à février, pendant la période de saison sèche où les densités de populations de $Z$. variegatus sont élevées dans l'ensemble de la zone, contrairement à celles de la saison pluvieuse. Les densités observées à cette période pluvieuse sont négligeables. Les épizooties ont été importantes au cours des saisons 1995-1996 et 1996-1997. Pendant la saison 1996-1997, la prévalence de l'infection a atteint au maximum $10 \%, 14 \%, 24 \%$ et $46 \%$ respectivement à Agbata, Lama 1, Lama 3 et Lama 2. Entre novembre 1996 et début janvier 1997, le taux moyen d'infection a varié entre 4 et 10\%, notamment à Agbata et Lama 3, mais était supérieur à 15\% à Lama 2. En 1997-98, les épizooties étaient très faibles, variant entre 0 et $2 \%$ sur la plupart des sites, sauf à Hinvi où elles ont atteint $14 \%$. Parmi les facteurs abiotiques examinés, à savoir la température, la pluviométrie, l'humidité relative et la radiation solaire, cette dernière est apparue comme le principal facteur ayant régulé les épizooties au cours des trois saisons.
\end{abstract}

Mots clés: Zonocerus variegatus, Entomophaga grylli, Entomophthorales, épizootie.

\section{Abstract}

Factors affecting the occurrence of epizootics of Entomophaga grylli (Zygomycetes, Entomophthorales) on Zonocerus variegatus populations in Southern Benin in West Africa

The seasonal occurrence of Entomophaga grylli on Zonocerus variegatus populations was monitored in the four Southern departments of Benin. The study showed that E. grylli was one of the major mortality factors of grasshopper's larvae. Infections occurred principally from november to february during dry season when Z. variegatus populations are important. Densities observed at this period are generally high in the studied zones, contrary to those of wet season witch are negligible. Epizootics were important during 1995-96 and 1996-97 seasons. During 1996-97, prevalence of infection reached in maximum 10\%, 14\%, 24\% and $46 \%$ the 11 December at Agbata, Lama 1, Lama 3 and Lama 2 sites respectively. Between November 1996 and start January, infection mean rate varied from 4 to $10 \%$ notably at Agbata and Lama 3, but was more than 15\% at Lama 2. In 1997-98 epizootics were very few, between 0 and $2 \%$ on the majority sites, but not at Hinvi were a maximum of $14 \%$ was reached in 1997. Among abiotic factors examined, namely temperature, relative humidity and solar radiation, this last appeared to be the principal epizootics regulating factor.

Key words: Zonocerus variegatus, Entomophaga grylli, Entomophthorales, epizootic 


\section{Introduction}

Zonocerus variegatus (L.) est un criquet polyphage qui s'attaque à une large gamme de plantes cultivées ou sauvages dont le manioc. L'insecte est considéré dans beaucoup de pays de la zone humide d'Afrique comme un ravageur important (Chapman et al. 1986; Modder, 1994) même si son importance économique réelle n'est pas bien définie. La liste de ses plantes hôtes atteint 250 espèces (Chiffaud \& Mestre, 1990). De nombreuses études récentes tentent encore de comprendre certains aspects du cycle de vie de l'insecte, de même que ses préférences alimentaires (Le Gall et al., 1998 ; Douro-Kpindou et al. 2000 ; Messi et al ., 2006).

$Z$. variegatus est l'hôte de nombreux ennemis naturels incluant des bactéries, des protozoaires, des nématodes, le Diptère Sarcophagidae Blaesoxipha filipjevi et le champignon Entomophaga grylli. C'est un champignon Entomophthorale qui infecte naturellement certaines espèces acridiennes. II entraîne d'importantes épizooties. Elles ont été décrites par de nombreux auteurs en Afrique et ailleurs dans le monde (Roffey, 1968; Chapman \& Page, 1979).

Si $E$. grylli a été assez étudié chez les sauteriaux en Amérique du Nord (Soper et al., 1983; Carruthers et al., 1988), ce n'est pas le cas en Afrique où pourtant les épizooties sont fréquentes chez $Z$. variegatus chez qui il est considéré comme le principal facteur de mortalité (Chapman \& Page, 1979) .

Les travaux réalisés sur le couple $E$. grylli / $Z$. variegatus en Afrique sont rares. Ce sont ceux de Hendrix (1943) rapportés par Chiffaud \& Mestre (1990) et ceux de Chapman \& Page (1979). II est donc clair que beaucoup reste à faire à ce sujet. II semble que les épizooties sont généralement fonction de l'humidité. La présente étude tente d'élucider la corrélation entre les variables climatiques et l'incidence de la maladie chez $Z$. variegatus.

\section{Matériel et Méthodes}

\subsection{Sites d'étude}

Les prospections ont été effectuées dans les quatre départements du Sud du Bénin sur plusieurs sites. Ce sont les départements (ou Provinces) du Mono, de l'Atlantique, de l'Ouémé et du Zou (Fig. 1). La superficie échantillonnée par site est de 1 ha. Les sites ont été géoréférencés grâce à un appareil GPS de marque Magellan, modèle GPS Nav 5000 DX ${ }^{\mathrm{TM}}$. II s'agit des sites suivants:

- trois sites dans la forêt de Lama, dans le département du Zou, désignés respectivement par Lama 1, Lama 2 et Lama 3 dont les coordonnées géo-graphiques sont dans l'ordre: Lama 1 ( $6^{\circ} 57,821 \mathrm{~N}$ et $\left.2^{\circ} 10,658 \mathrm{E}\right)$, Lama $2\left(6^{\circ} 58,263 \mathrm{~N}\right.$ et $2^{\circ}$ $10,750 \mathrm{E})$ et Lama $3\left(6^{\circ} 58,811 \mathrm{~N}\right.$ et $2^{\circ}$ $07,460 \mathrm{E})$

- deux sites dans le département de l'Atlantique au sud (zone d'Allada): Agbata de coordonnées $6^{\circ} 49,490 \mathrm{~N}$ et $2^{\circ} 20,236$ E et Hinvi $6^{\circ} 45,920 \mathrm{~N}$ et $2^{\circ} 10$, $114 \mathrm{E}$.

- deux sites dans le département de l'Ouémé à l'ouest; un dans la localité de Ita-djébou $\left(6^{\circ} 50,380 \mathrm{~N}\right.$ et $\left.2^{\circ} 10,330 \mathrm{E}\right)$ et l'autre à Sakété $\left(6^{\circ} 46,476 \mathrm{~N}\right.$ et $2^{\circ} 40,447$ E).

Ces sites ont été retenus en raison des densités importantes de criquets. La flore sur ces sites est constituée principalement par du maïs (Zea mays L.), avec la présence parfois de manioc (Manihot esculenta Crantz). La jachère autour des champs est dominée par Chromolaena odorata L. et certaines graminées sauvages comme Imperata cylindrica L. ainsi que d'autres mauvaises herbes communes. 


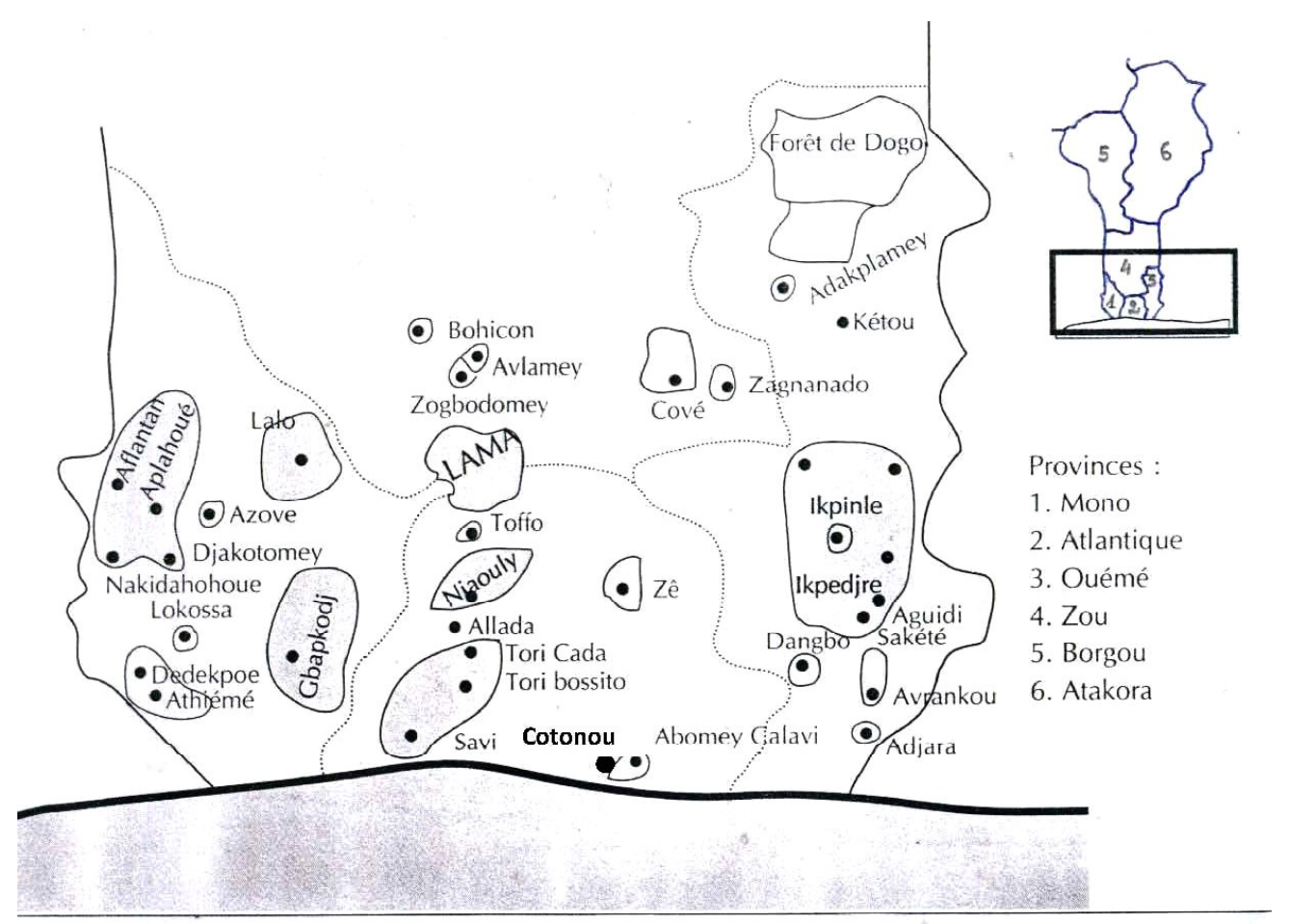

Figure 1: Carte du Bénin présentant les zones d'études (Paraiso et al., 1992), modifié

\subsection{Estimation des densités}

L'estimation des densités par site se fait en comptant le nombre de Zonocerus variegatus présents par surface de $1 \mathrm{~m}^{2}$ incluant le sol et la végétation, à raison de 25 surfaces par site (Lecoq \& Mestre, 1988; Douro-Kpindou et al., 1995). Ces surfaces, de forme carrée, sont distantes d'au moins $5 \mathrm{~m}$ suivant une ligne quelconque. Le nombre moyen d'insectes par $\mathrm{m}^{2}$ représente la densité.

\subsection{Estimation du taux d'infection}

Cinquante (50) criquets, tous stades confondus, sont collectés par site une fois par semaine ou toutes les deux semaines suivant l'abondance des insectes. Les criquets collectés sur les sites sont conservés au laboratoire dans des bouteilles en plastique de $1,5 \mathrm{~L}$ de volume, par lots de 25 individus, pendant un mois et nourris quotidiennement avec des feuilles de manioc. Le fond des bouteilles est coupé et remplacé par une toile. Chaque jour, le nombre d'insectes morts est noté et la cause de la mort recherchée. Les insectes tués par $E$. grylli se reconnaissent par la sporulation du champignon ou par des symptômes caractéristiques. En cas de doute, les insectes morts sont mis en observation pendant $24 \mathrm{~h}$ avant d'être incubés sur du coton hydrophile mouillé durant $48 \mathrm{~h}$ pour favoriser la sporulation. Au besoin, des prélèvements sur certains insectes sont observés au microscope pour rechercher la présence des organes du champignon (protoplastes, corps hyphaux, hyphes, conidiophores et conidies).

\subsection{Analyse des facteurs abiotiques}

La température et l'humidité relative $(\mathrm{HR})$ ont été relevées directement sur le site d'Agbata à l'aide d'un thermo-hygromètre mécanique. La fiche d'enregistrement est renouvelée chaque semaine. Les données pluviométriques ont été obtenues à l'aide de pluviomètres cylindriques simples à partir des stations météo les plus 
proches (Agbata, Cotonou, Lama 1 et 3). Les données sur la radiation solaire, grâce à un pynanographe, model R401, ont été fournies par le service météo de la station IITA Cotonou, de même que la température et l'humidité relative à Cotonou (thermomètre model EL 504-016, thermo-hydrographe model 8368-00). Les observations ont surtout été intensives de novembre à mars, période où les épizooties à E. grylli sont généralement observées, de 1996 à début 1998.

\subsection{Analyse statistique des données}

Une analyse de régression multiple suivant le programme «G 5 program stepwise multiple regression analysis» a été réalisée en considérant le facteur site comme facteur variable. Le pourcentage d'infection a été transformé suivant la formule arcsin. Le pourcentage d'infection transformé pour chaque date et par site a été régressé contre tous les autres facteurs: la pluviométrie en $\mathrm{mm}$, la température en ${ }^{\circ} \mathrm{C}$, l'humidité relative en \% et la radiation solaire transformée en sa racine carrée pour chaque date, avec ou sans la densité.

\section{Résultats}

\subsection{Variation de la densité des populations du criquet dans le temps}

Les densités ont été observées durant les saisons $1996 / 1997$ et $1997 / 1998$

A Lama 1, les fluctuations de densités étaient plus marquées, avec deux pics: un pic de 8,5 insectes. $\mathrm{m}^{-2}$ en novembre 1996 et un autre de près de 9 insectes. $\mathrm{m}^{-2}$ en février-mars 1997 (Fig. 2). En 1997 / 1998 pour la même période, peu d'insectes étaient présents sur ce site et de ce fait, la densité n'a pas été estimée; contrairement à Lama 2 où elle a varié entre 11 et 4 insectes. $\mathrm{m}^{-2}$ de décembre 1997 à janvier 98 et 1,8 insectes. ${ }^{-2}$ en février (Fig. 3). A Lama 3, les densités étaient également élevées: entre 23 et 10 insectes, de novembre à mi-décembre, et de 13 à 10 insectes. $\mathrm{m}^{-2}$ entre fin février et la mimars. En dehors de ces deux pics, la densité a varié entre 0 et 7 insectes. $\mathrm{m}^{-2}$ (Fig. 4). En 97/98, la population était très faible. Les pics de populations correspondent respectivement aux jeunes stades larvaires et à la période d'accouplement et de ponte chez les adultes, aussi bien à Lama 1 qu'à Lama 3.

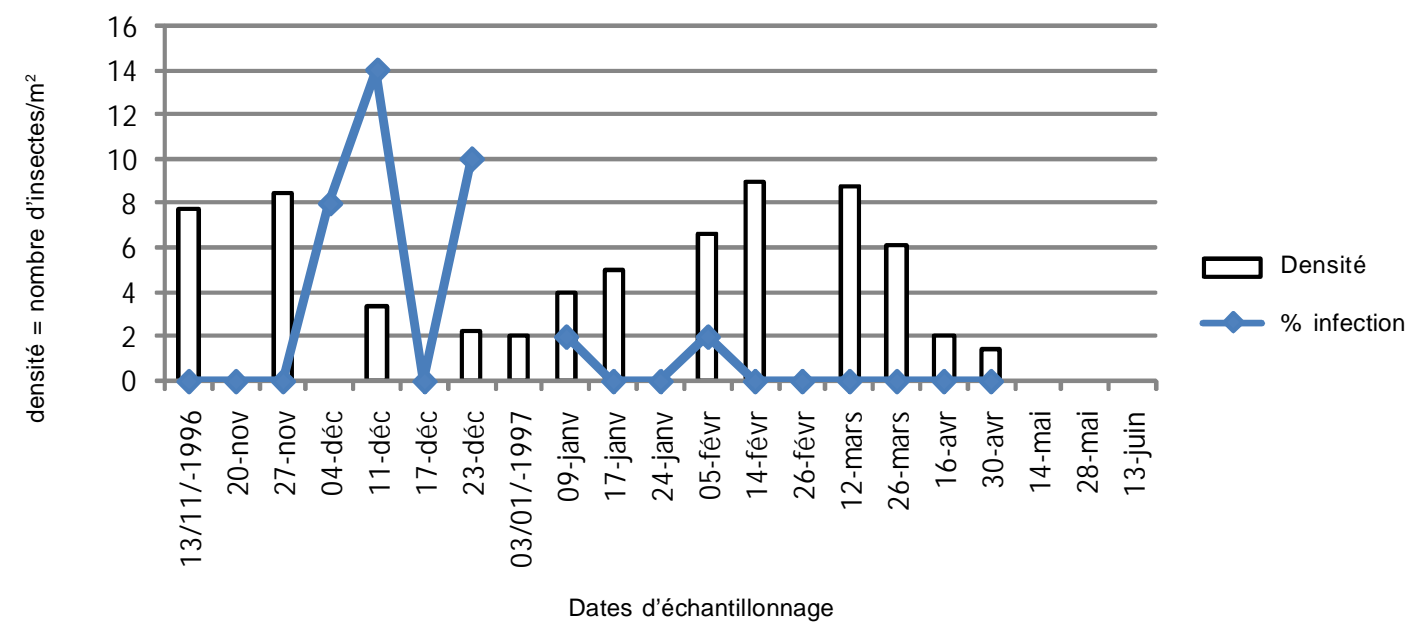

Figure 2 : Prévalence de E. grylli chez $Z$. variegatus à Lama 1 


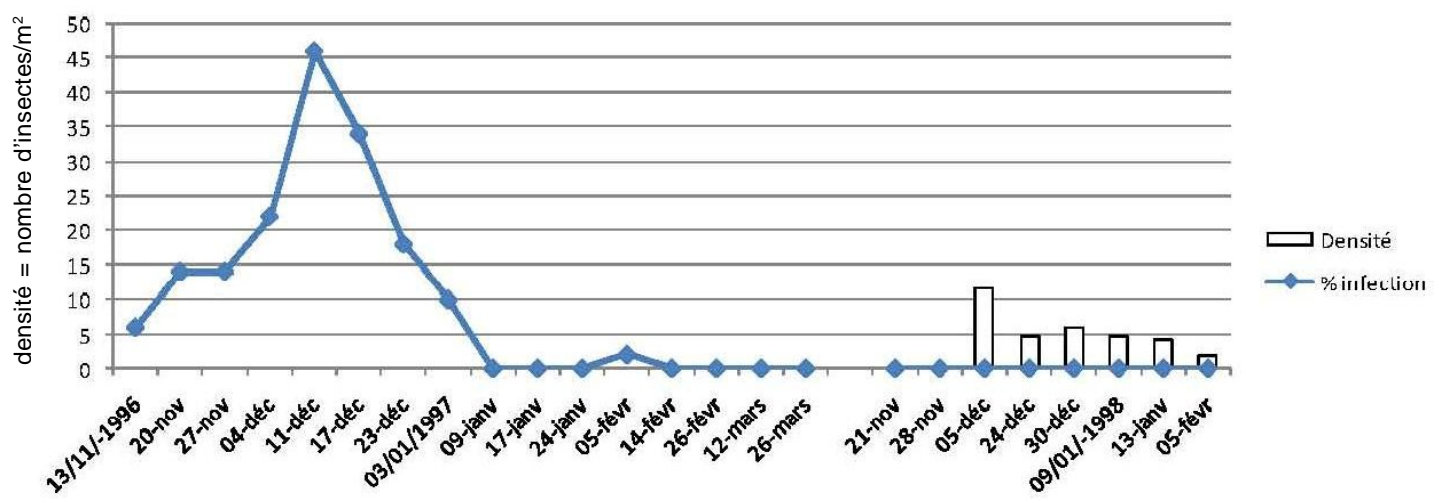

Dates d'échantillonnage

Figure 3 : Prévalence de E. grylli chez Z. variegatus à Lama 2

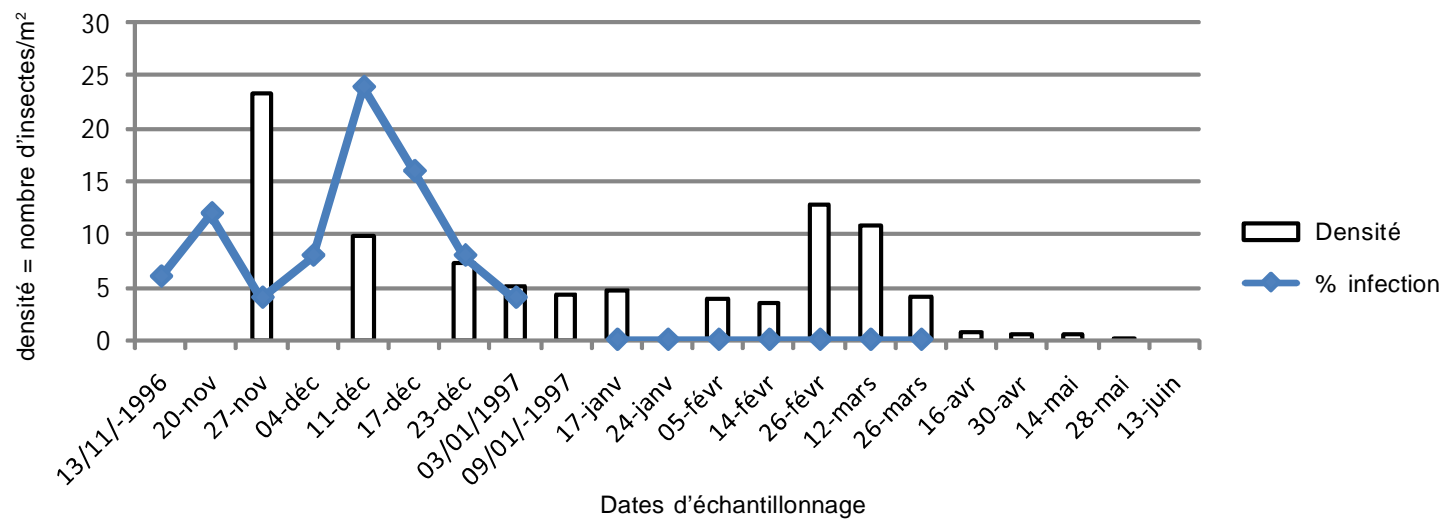

Figure 4 : Prévalence de E. grylli chez Z. variegatus à Lama 3

A Agbata, les densités étaient plus importantes entre novembre 96 et début janvier 97 et ont varié entre 13 et 5 insectes. $\mathrm{m}^{-2}$. Entre mi-janvier et mi-mars l'on comptait entre 4 et 2 insectes. $\mathrm{m}^{-2}$; puis moins d'un insecte en mai-juin et aucun insecte en juillet et Août. En 97 / 98 les densités étaient plus faibles au cours de la même période : entre 8 et 2 insectes.m ${ }^{-2}$ (Fig. 5).

A Ita-djébou et Sakété, les densités étaient moins importantes que celles des sites précédents pour la même période (Fig. 6 et 7). Les populations de criquets étaient plus abondantes en 96/97 qu'en 97/98 sur les différents sites à l'exception de Hinvi (Fig. 8) où elles étaient importantes en $97 / 98$. La plus grande population de criquets s'observe pendant la saison sèche, de novembre à février. 


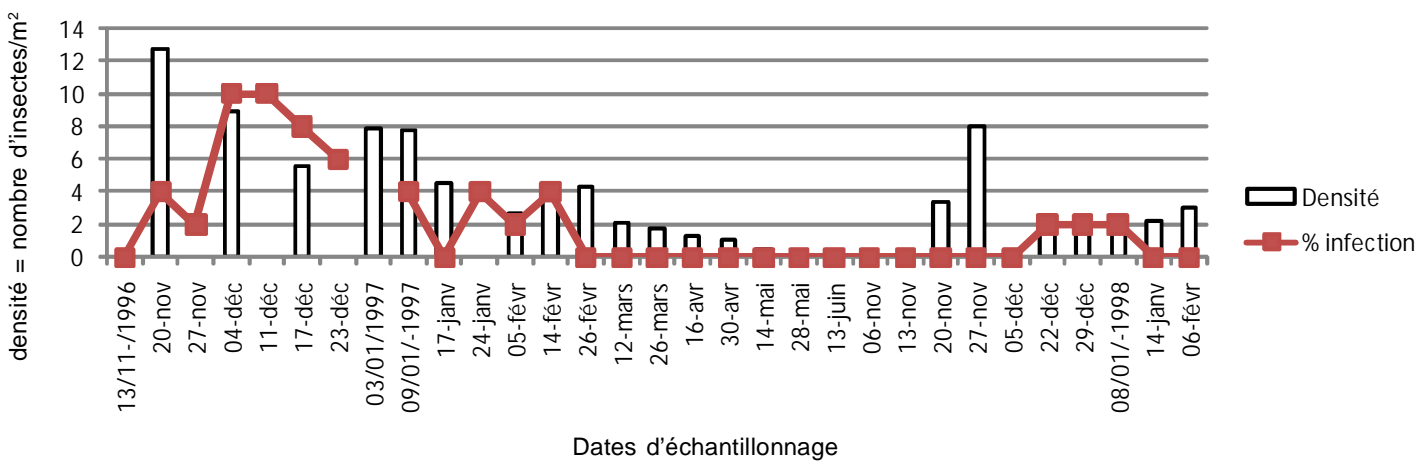

Figure 5 : Prévalence de E. grylli chez Z. variegatus à Agbata

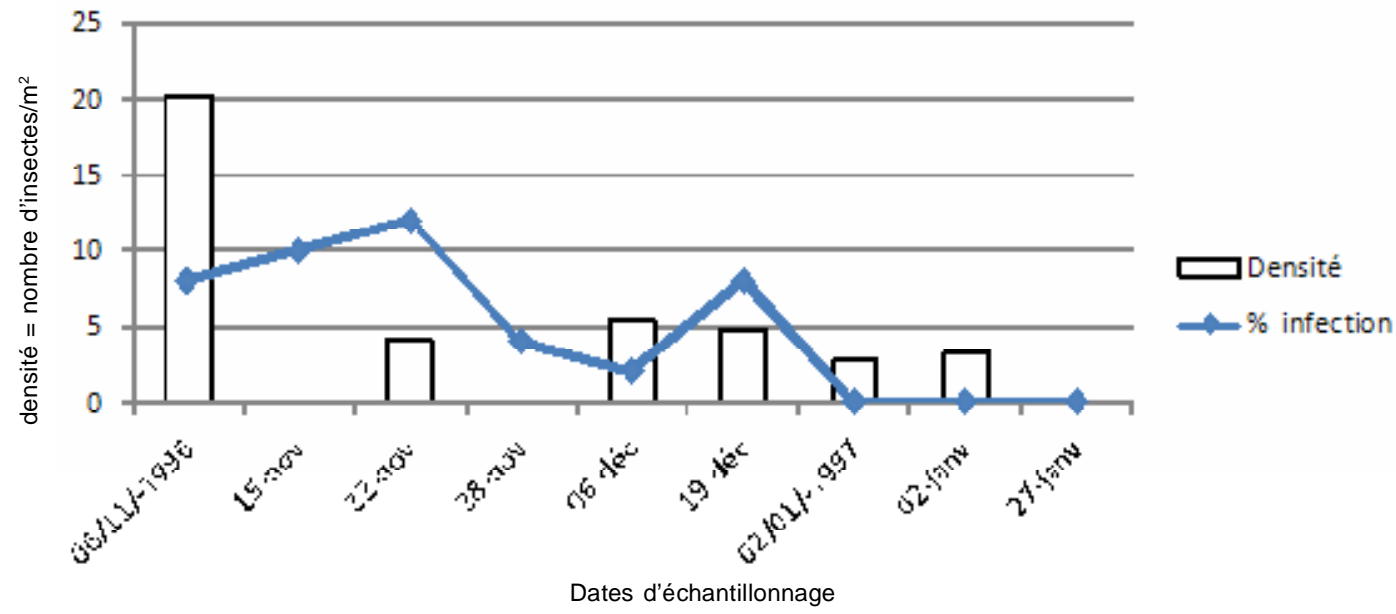

Figure 6 : Prévalence de E. grylli chez Z. variegatus à Ita-Djébou

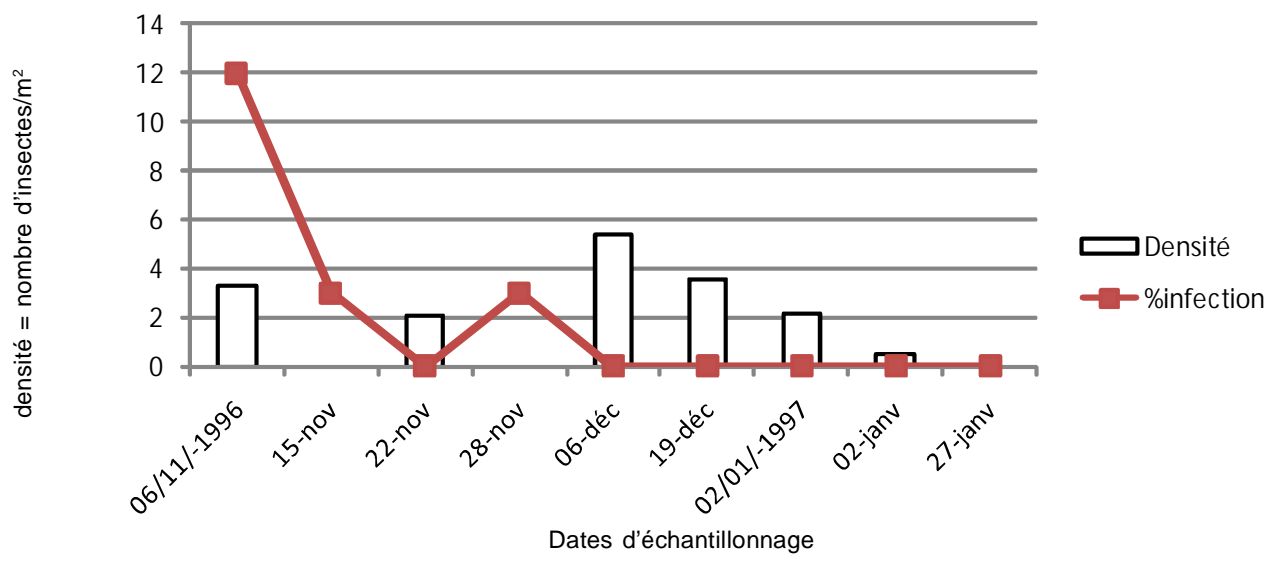

Figure 7 : Prévalence de E. grylli chez Z. variegatus à Sakété 


\subsection{Prévalence et impact du champignon sur les populations de $Z$. variegatus}

\subsubsection{Saison 1995 / 1996}

Avant le début des prospections, des épizooties à E. grylli ont été observées dans le Mono, notamment en janvier et février. Une épizootie extrêmement sévère a été observée à la fin du mois de décembre et au mois de janvier sur la station IITA Cotonou dans une parcelle de manioc, décimant presque entièrement la population locale de $Z$. variegatus.

\subsubsection{Saison 1996 / 1997}

Les premières infections ont été observées vers la fin du mois d'octobre (Figs. 2 à 8). Les infections ont eu lieu essentiellement en novembre et décembre. L'infection a été plus importante sur le site de Lama 2 où elle a atteint $46 \%$. La prévalence de l'infection sur ce site est restée proche ou supérieure à $15 \%$ entre le 20 novembre 1996 et le 3 janvier 1997 (Fig. 3). L'infection était relativement importante à Lama 3 avec un taux d'infection de 24\% le 11 décembre (Fig. 4).
Sur les autres sites d'étude, les infections ont été plus faibles avec des valeurs maximales variant entre 10 et $14 \%$. C'est notamment le cas des sites de Lama 1 et Agbata (Figs. 2 et 5), de Ita-djébou et Sakété (Figs. 6 et 7). Entre fin décembre et début janvier, une chute importante de l'infection est observée sur la plupart des sites. Elle passe de 10 à $2 \%$, de 18 à $0 \%$ et de 6 à $4 \%$ respectivement à Lama 1 et 2 et Agbata du 23/12 au 9/01. L'infection reste très faible et varie de 0 et $4 \%$ du 17 janvier au 5 février 97 sur l'ensemble des sites. A partir du 26 février, on n'observe plus aucune infection $(0 \%)$ sur tous ces sites en dépit de la présence d'insectes adultes en nombre encore mportant.

\subsubsection{Saison 1997 / 1998}

Les prospections effectuées sur l'ensemble des sites, dans les quatre départements ont mis en évidence l'absence d'infections notables dans toutes les zones visitées. S'agissant des sites d'échantillonnage, il n'y a pas eu d'infection sauf à Agbata avec un taux maximum de $2 \%$ et de $14 \%$ à Hinvi (Figs. 5 et 8)

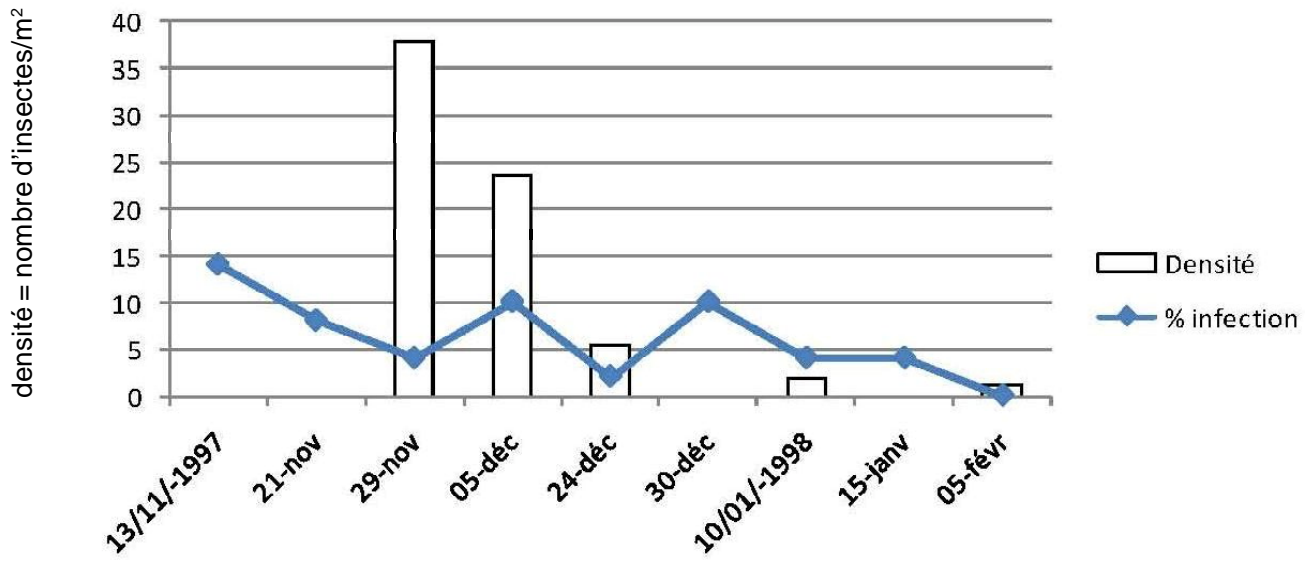

Dates d'échantillonnage

Figure 8 : Prévalence de E. grylli chez Z. variegatus à Hinvi

\subsection{Conditions climatiques}

Les températures relevées sur le site d'Agbata en $1996 / 1997$ et $1997 / 1998$ se présentent comme suit la température moyenne minimale hebdomadaire a varié entre 22 et $26^{\circ} \mathrm{C}$; la moyenne maximale entre 29 et $37^{\circ} \mathrm{C}$ et la moyenne hebdomadaire entre 26 et $31^{\circ} \mathrm{C}$. La température journalière reste généralement supérieure à $30^{\circ} \mathrm{C}$ pendant 4 à $8 \mathrm{~h}$. L'analyse des températures moyennes minimales, moyennes maximales et moyennes mensuelles à l'IITA Cotonou en 1995, 1996 et 1997 indique des intervalles de variations respectives de $22-24^{\circ} \mathrm{C}, 27-33^{\circ} \mathrm{C}$ et $24-29^{\circ} \mathrm{C}$. 
L'humidité relative moyenne maximale, moyenne minimale et moyenne hebdomadaire enregistrée à Agbata entre décembre 1996 et mai 1997 a varié respectivement entre $95-98 \%, 23-58 \%$ et $60-77 \%$. L'HR a été supérieure à $90 \%$ pendant en moyenne $12 \mathrm{~h}$ par jour sauf en février où cette période était inférieure à $10 \mathrm{~h}$ par jour (Fig. 9). Elle est restée supérieure ou égale à $95 \%$ pendant au moins $6 \mathrm{~h}$ par jour et par semaine à l'exception de la période du 12 au 16 décembre et du mois de février. Les moyennes mensuelles minimales, maximales, et moyennes mensuelles à la station IITA/ Cotonou en 1995, 96 et 97 ont varié respectivement comme suit: $41-80 \%, 92-98 \%$ et $69-89 \%$.

Les données pluviométriques montrent que les mois les plus secs sont novembre, décembre, janvier et février sur tous les sites de 95 à 97 (Fig. 10). La pluviométrie en octobre 1997 était exceptionnellement élevée sur tous les sites (entre 150 et $350 \mathrm{~mm}$ ) (Fig. 10).
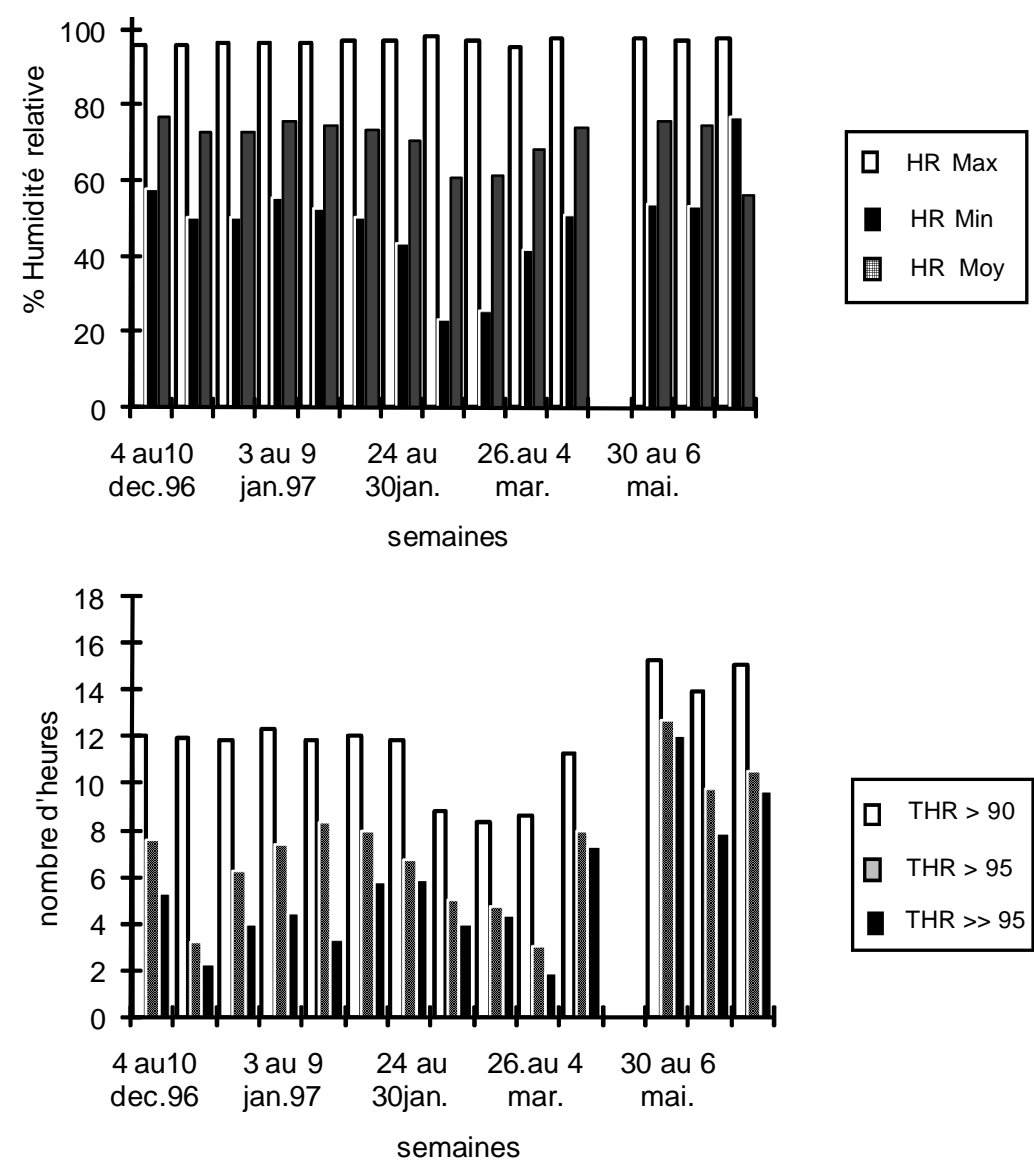

Figure 9: Humidité relative moyenne journalière hebdomadaire (au dessus) et nombre d'heures où elle est supérieure ou égale à 90\%, 95\% et strictement supérieure à 95 \% (en dessous) sur le site d'Agbata en 1996/1997

HRmax: humidité relative moyenne maximale

HRmin: humidité relative moyenne minimale

HRmoy: humidité relative moyenne mensuelle

$T H R \geq 90$ : temps où HR est $\geq 90 \%$

$T H R \geq 95$ : temps où $H R$ est $\geq 95 \%$

THR > 95: temps où HR est $>95 \%$ 


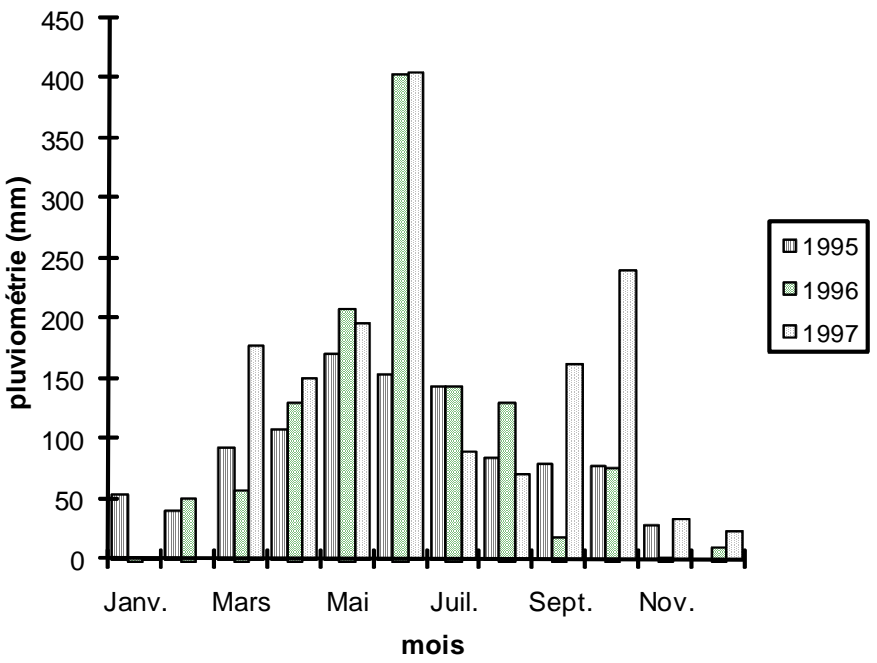

Figure 10 : Pluviométrie à la station IITA Cotonou en 1995, 1996 et 1997 (Source service météo IITA / Cotonou

La radiation solaire moyenne journalière entre septembre et février était inférieure à $300 \mathrm{~g} \mathrm{cal.cm}$ ${ }^{2}$ jour ${ }^{-1}$ en $1995 / 1996$ et en 1996/1997 sauf en janvier et février 1997 où elle était supérieure à $350 \mathrm{~g} \mathrm{cal.cm}^{-2} \mathrm{jour}^{-1}$. En 1997/98, elle était au

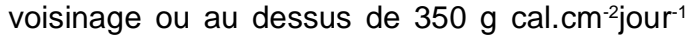
tous ces mois (Tableau 1). Le nombre de jours par mois où la radiation solaire moyenne

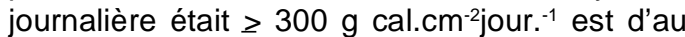
moins 20 jours pour tous les mois considérés en
$1997 / 1998$ mais d'au plus cinq jours en $95 / 96$ sauf en octobre. II en est de même en $96 / 97$ sauf en janvier et février 97 (Tableau 1).

L'analyse de régression multiple suivant le programme «GENSTAT» 5 program stepwise multiple regression analysis" montre une corrélation (négative) significative de coefficient 1,256 et une probabilité de 0,008 entre la radiation solaire et le pourcentage d'infection pour tous les sites confondus (Tableau 2).

Tableau 1 : Evolution de la radiation solaire entre septembre et février au cours des saisons 1995/1996, 1996/1997 et 1997/1998 à la station IITA Cotonou (source météo, IITA Cotonou)

a) Radiation solaire moyenne journalière ( $\mathrm{g}$-cal $/ \mathrm{cm}^{2} / \mathrm{jour}$ )

\begin{tabular}{ccccccc}
\hline & Septembre & Octobre & Novembre & Décembre & Janvier & Février \\
\hline $1995 / 1996$ & 261 & 288 & 271 & 237 & 241 & 265 \\
$1996 / 1997$ & 223 & 224 & 251 & 235 & 349 & 391 \\
$1997 / 1998$ & 377 & 392 & 392 & 338 & 327 & 372 \\
\hline
\end{tabular}

b) Nombre de jours/mois où la radiation solaire moyenne journalière est supérieure à $250 \mathrm{~g} \mathrm{cal} / \mathrm{cm}^{2} / \mathrm{jour}$

\begin{tabular}{lccccc}
\hline & Octobre & Novembre & Décembre & Janvier & Février \\
\hline $1995 / 1996$ & 26 & 22 & 10 & 11 & 19 \\
$1996 / 1997$ & 17 & 14 & 12 & 29 & 28 \\
$1997 / 1978$ & 26 & 21 & 25 & 25 & 28 \\
\hline
\end{tabular}

c) Nombre de jours/mois où la radiation solaire moyenne journalière est supérieure à $300 \mathrm{~g} \mathrm{cal} / \mathrm{cm}^{2} / \mathrm{jour}$

\begin{tabular}{lccccc}
\hline & Octobre & Novembre & Décembre & Janvier & Février \\
\hline $1995 / 1996$ & 13 & 4 & 0 & 0 & 5 \\
$1996 / 1997$ & 1 & 0 & 0 & 26 & 27 \\
$1997 / 1978$ & 20 & 20 & 23 & 24 & 28 \\
\hline
\end{tabular}


Tableau 2: Résultat de l'analyse statistique

a) Estimations des coefficients de la régression multiple

\begin{tabular}{lcccc}
\hline Variables & $\begin{array}{l}\text { Coefficients } \\
\text { de régression }\end{array}$ & Erreur standard & $\mathrm{t}(47)$ & $\mathrm{t}$ pr. \\
\hline Constante & 3 & 24 & 0,13 & 0,901 \\
$\%$ HR & 0 & 0,105 & 0,00 & 1,000 \\
Pluie $(\mathrm{mm})$ & $-1,31$ & 1,40 & $-0,93$ & 0,357 \\
VRadiation solaire & $-1,256$ & 0,452 & $-2,78$ & 0,008 \\
Température & 1,053 & 8,815 & 1,29 & 0,203 \\
\hline
\end{tabular}

b) Analyse de la variance du modèle de régression

\begin{tabular}{llllll}
\hline Variables de variation & DDL & SCE & CM & F c & F pr. \\
\hline \% HR & 1 & 67,26 & 67,26 & 2,18 & 0,146 \\
Pluie $(\mathrm{mm})$ & 1 & 112,95 & 112,95 & 3,66 & 0,062 \\
VRadiation solaire & 1 & 216,11 & 216,11 & 7,01 & 0,011 \\
Température & 1 & 51,50 & 51,50 & 16,07 & 0,203 \\
Erreur résiduelle & 47 & 1448,91 & 30,83 & & \\
Total & 51 & 1896,72 & 37,19 & & \\
\hline
\end{tabular}

\section{Discussion}

Les observations ont montré que l'infection à $E$. grylli est un facteur important de mortalité des larves du criquet $Z$. variegatus comme l'avaient rapporté par ailleurs Chapman \& Page (1979). Les taux d'infection naturelle à $E$. grylli sont plus importants, comparés à ceux dus à Metarhizium flavoviride sur les acridiens dans la même zone d'étude estimés en moyenne entre 1,8 et $2,9 \%$ selon le site (Shah et al., 1994).

Les infections à $E$. grylli sont plus importantes chez les larves âgées du criquet que chez les plus jeunes larves et les adultes. Cela pourrait s'expliquer par le fait que la densité de l'inoculum peut être supposée faible au moment de l'émergence des premiers stades du criquet. En outre, la quantité de spores chez les jeunes larves est plus faible du fait de leur petite taille. Enfin, ces jeunes larves sont souvent peu mobiles et regroupées sur les plantes, notamment de Chromolaena odorata. Un tel comportement pourrait limiter leur contact avec l'inoculum infectieux, contrairement aux stades avancés qui ont une plus grande propension à la dispersion. Dans une étude sur la cicadelle Empoasca kraemeri et l'Entomophthorale Zoophthora radicans, Galaini-Wraight et al. (1991) étaient parvenus à des observations similaires.
Des tests de laboratoire ont permis d'étudier l'infection artificielle du criquet, la sporulation, la germination des spores du champignon ainsi que le développement de la mycose. Une sensibilité plus faible chez les adultes que chez les larves âgées est suspectée (Gnago et al., 2004).

II n'a pas été établi de relation directe entre la mycose et la densité des criquets. Le fait que les éclosions soient échelonnées dans le temps (Douro-Kpindou et al., 2000) peut compenser la mort des insectes. Par ailleurs la densité étant élevée sur tous les sites (ces sites ayant été choisis en raison des fortes populations du criquet), l'effet densité devient négligeable. Les infections étaient présentes ou absentes indépendamment des densités.

Le fait que les criquets aient des comportements à la fois grégaires à certaines périodes ou migratoires à d'autres ne facilite pas la compréhension du rapport mycose/densité des criquets, surtout chez Z. variegatus chez lequel plusieurs facteurs entraînent des mouvements de population (évolution des stades, reproduction, disponibilité des plantes alimentaires). Quoi qu'il en soit, la densité de la population hôte est un facteur jugé important dans le développement des épizooties tout comme la densité de l'inoculum du pathogène (Tanada \& Fuxa, 1987; Galaini-Wraight et al., 1991). 
La variation de la température d'un jour à l'autre ou d'une année à l'autre a été faible et ne saurait expliquer le faible niveau d'infection enregistré, particulièrement en 1997/98. II est connu que certains acridiens élèvent la température de leurs corps à celle optimale à leur développement. Un tel phénomène peut réduire la mycose en leur sein (Carruthers et al., 1992).

Chapman et al. (1986) soupçonnent l'existence de thermorégulation chez $Z$. variegatus. Une telle thermorégulation peut minimiser l'effet de la variation de leur température interne au cours de la journée.

Le développement des infections à $E$. grylli a été souvent associé à la pluie (Roffey, 1968; Chapman \& Page, 1979; Chapman et al. 1986). C'est également le cas pour d'autres mycoses. La pluie agirait en favorisant la germination des spores de résistance présentes dans les cadavres secs d'insectes ou dans le sol (Weseloh \& Andreadis, 1992). Au Sud du Bénin, les jeunes larves de $Z$. variegatus sont déjà abondantes en octobre, mois au cours duquel les pluies sont suffisamment importantes pour initier les premières infections. L'humidité relative est par la suite suffisante pour perpétuer l'infection. La pluie ne serait donc pas un facteur limitant de 1995 à 1997.

Tout comme la pluie, l'humidité relative n'a pas été un facteur limitant puisque les valeurs quotidiennes enregistrées étaient supérieures ou égales à $95 \%$ pendant au moins $6 \mathrm{~h}$, sauf en février et mars. Ce qui est suffisant pour créer des conditions de saturation à la surface des feuilles et des insectes, conditions favorables à la germination des spores. En effet, plus de $50 \%$ des spores du champignon germent, à $100 \%$ d'humidité relative à 20 ou $25^{\circ} \mathrm{C}$, au bout de $4 \mathrm{~h}$ de temps (Gnago et al., 2004).

La radiation solaire apparaît comme le facteur le plus significatif pour mieux expliquer la variation de la prévalence à $E$. grylli parmi les populations de $Z$. variegatus au cours des saisons 1995/96, 96/97 et 97/98. En effet, les infections à $E$. grylli ont été importantes au cours des deux premières saisons. Ceci coïncide avec des radiations moyennes journalières inférieures à $300 \mathrm{~g} \mathrm{cal.cm}^{-2} \mathrm{jour}^{-1}$ de septembre à février en 95/96 et de septembre à décembre en $96 / 97$. En $97 / 98$, où ces radiations moyennes quotidiennes étaient nettement supérieures à 300 voire à $350 \mathrm{~g} \mathrm{cal} \mathrm{cm}^{-2} \mathrm{jour}^{-1}$ de septembre à février, les épizooties ont été plutôt rares. Cela est encore évident si l'on considère la durée de

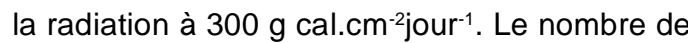
jours par mois où la radiation a dépassé $300 \mathrm{~g}$ cal. $\mathrm{cm}^{-2}$ jour ${ }^{-1}$ était inférieur ou égal à 5 , de novembre à février $95 / 96$ et en octobre, novembre et décembre 96. Pendant ce temps, les épizooties étaient importantes. Par contre, lorsque les épizooties n'étaient pas importantes (janvier et février 97 et saison 97/98), la radiation est restée à $300 \mathrm{~g} \mathrm{cal.cm}^{-2} \mathrm{jour}^{-1}$ pendant au moins 20 jours par mois (Tableau 1 ).

Cet effet de la radiation semble plus évident si l'on tient compte du comportement des individus infectés peu avant leur mort. En effet, $Z$. variegatus infectés par $E$. grylli grimpent aux sommets des tiges et des feuilles avant de mourir. Ceci les expose plus aux radiations solaires avec comme conséquence une mortalité des spores et une réduction de l'inoculum.

Des études au laboratoire et en champ ont montré l'effet des radiations solaires sur la mortalité des conidies. Celle-ci a atteint 100\% chez Entomophthora muscae (Carruthers \& Haynes, 1986) et chez E. grylli (Carruthers et al., 1988), en fonction de l'intensité et de la durée d'exposition aux radiations.

Enfin, il ne faut pas perdre de vue que l'environnement est un ensemble complexe où tous les facteurs interagissent. L'importance d'autres facteurs tels que le microclimat, le type de couvert végétal, l'influence du pH et l'aération du sol est à prendre en compte.

La forme sous laquelle survit $E$. grylli d'une saison à l'autre chez Z. variegatus n'est pas connue. Deux hypothèses semblent probables:

$1 /$ le champignon survit in vivo, soit à l'intérieur des populations de $Z$. variegatus, soit sur d'autres hôtes alternatifs. Le fait que $Z$. variegatus disparaisse complètement sur certains sites alors que la maladie y est présente plus tard accrédite plus l'hypothèse des hôtes alternatifs.

2/ le champignon passe les périodes difficiles sous forme de spores de résistance, soit dans les cadavres secs d'insectes soit dans le sol. 
Nous avons en effet observé des spores de résistance d'E. grylli chez $Z$. variegatus. La seconde hypothèse nous semble la plus probable, même si les deux hypothèses sont plausibles.

\section{Conclusion}

E. grylli est un facteur important dans la régulation naturelle des populations de $Z$. variegatus. L'infection a atteint $46 \%$ sur l'un des sites et dépassé $10 \%$ sur les autres. Cette étude montre l'importance de la radiation solaire dans la régulation des épizooties à $E$. grylli chez $Z$. variegatus au sud du Bénin. L'intensité et la durée de la radiation semblent influencer négativement les épizooties. Les autres facteurs comme la température, la pluie et l'humidité relative ne sont pas apparus comme des facteurs limitant pour les épizooties. Une telle étude peut contribuer à une meilleure compréhension des mécanismes de développement des épizooties naturelles à $E$. grylli en zone tropicale et permettre aussi de déterminer les conditions d'introduction et donc de succès d'épizooties artificielles dues à ce champignon en matière de lutte biologique. Cependant, la maîtrise en amont de l'infection artificielle des insectes au laboratoire est indispensable, de même que l'identification de la forme de conservation et de survie du champignon dans la nature.

\section{Remerciements}

Nous dédions cette étude à la mémoire de John Christopher LOMER, anciennement à l'Institut International d'Agriculture Tropicale (IITA Cotonou Bénin, qui a contribué à la réalisation de ce travail.

Nous remercions I'UA (I'Union Africaine, exOUA) pour son soutien financier dans le cadre d'une thèse de Doctorat, ainsi que I'IITA qui a mis à notre disposition les moyens logistiques.

\section{Références citées}

Carruthers, R. I. \& Haynes, D. L. 1986. Temperature, moisture, and habitat effects on Entomophthora muscae (Entomo- phthorales: Entmophthoraceae) conidial germination and survival in the onion agroecosystem. Environ. Entomol., 15, 11541160.

Carruthers, R.I., Feng, Z., Ramos, M. E. \& Soper, R. S. 1988. The effect of solar radiation on the survival of Entomophaga grylli (Entomphthorales: Entomophthoraceae) conidia. J. Invertebr Pathol., 52, 154-162.

Carruthers, R. I., Larkin T. S. \& Firstencel, H. 1992. Influence of thermal ecology on the mycosis of a rangeland grasshopper. Ecologie., 73 (1): 190-204.

Chapman, R. F.\& Page, W. W. 1979. Factors affecting the mortality of the grasshopper Zonocerus variegatus, in southern Nigeria. J. Anim. Ecol., 48, 271-288.

Chapman, R. F., Page W.W. \& Mccaffery, A. R. 1986. Bionomics of the variegated grasshopper (Zonocerus variegatus) in West and Central Africa. Ann. Rev. Entomol., 31, 479-505.

Chiffaud, J. \& Mestre, J. 1990. Le criquet puant Zonocerus variegatus (Linné, 1758). Essai de synthèse bibliographique. CIRADPRIFAS. Département GERDAT 138 p.

Douro-Kpindou, O.-K, Godonou, I., Houssou, A., Lomer, C. J. et Shah, P. A. 1995. Control of Zonocerus variegatus by ultra-low volume application of an oil formulation of Metarhizium flavoviride conidia. Biocontrol Sci. and Technol., 5, 131-139.

Douro-Kpindou, O.-K, Lomer, C. J., Langewald, J. \& Bocco, Y. 2000. Cycle biologique et durée des stades larvaires du croquet puant Zonocerus variegatus (Linné, 1758) (Orthopthera, Pyrgomorphidae) au sud du Bénin. Insect Sci. Appl. 20(2):109-116.

GALAI Galaini-Wraight, S., Wraight, S. P., Carruthers, R. I., Magalhaes, B. P. et Roberts,

D. W. 1991. Description of Zoophthora radicans (Zygomycetes: Entomophthoraceae) epizootic in a population of Empoasca kraemeri (Homoptera: Cicadellidae) on beans in central Brazil. J. Invertebr. Pathol., 58, 311-326.

Gnago, A. J., Foua Bi, K. \& Lomer, J.C. 2004. Effet de la température et de l'humidité relative sur le développement de la mycose 
à Entomophaga grylli Batko (Zygomycètes, Entomophthorales) chez Zonocerus variegatus L. (Orthoptera, Pyrgomorphidae). Agron. Afr. 16 (2): 19-31.

Lecoq, M. \& Mestre, J. 1988. La surveillance des sautériaux du Sahel. Collection Acridologie opérationnelle $\mathrm{N}^{\circ} 2$ CIRAD-PRIFAS, Montpellier.

Le Gall, P., Mingouolo, E. \& Bani, G. 1998. Diet of Zonocerus variegatus (L.) (Orth., Acridiae) in cassava fields in Congo. J. Appl. Ent., 122, 9-13.

Messi, J., Kekeunou, S. \& Weise, S. 2006. Abundance and life cycle of Zonocerus variegatus (Orthopthera: Pyrgomorphidae) in the humid forest zone of southern Cameroon. Entomol. Sci. 9, 23-30.

Modder, W. W. D. 1994. Control of the variegated grasshopper, Zonocerus variegatus (L.) on cassava. Afr. crop Sci. J., 2 (4) 391-406.

Paraïso, A., Lomer, C.J., Godonou, I. \& Kpindu,, D. 1992. Etudes préliminaires sur l'écologie de Zonocerus variegatus au Bénin. In Lomer, C. J. \& Prior, C., Eds. Lutte biologique contre les acridiens. Compte rendu d'un atelier à l'IITA, Cotonou, Bénin, 29 avril-1 mai, 1991. C.A.B International, pp. 133-142.

Roffey, J. 1968. The occurrence of the fungus Entomophaga grylli Fresenius on Locusts and grasshoppers in Thailand. J. Invertebr. Pathol. 11, 237-241.

Shah, P. A., Godonou, I., Gbongboui, C. \& Lomer, C. J. 1994. Natural levels of fungal infections in grasshoppers in northern Benin. Biocontrol Sci. and Technol., 4, 331-341.

Soper, R. S., May, B. \& MartinelL, B. 1983. Entomophaga grylli enzyme polymorphism as a technique for pathotype identification. Environ. Entomol., 12, 720-723.

Tanada, Y. \& Fuxa, J.R. 1987. The pathogen population. In Fuxa, J.R. and Tanada, Y. eds. Epizootiology of insect diseases (. John Wiley and Sons, New York. pp. 113-157.

Weseloh, R. M. \& Andreadis, T. G. 1992. Epizootiology of the fungus Entomophaga maimaiga, and its impact on gypsy moth populations. J. Invertebr. Pathol, 59, 133-141. 